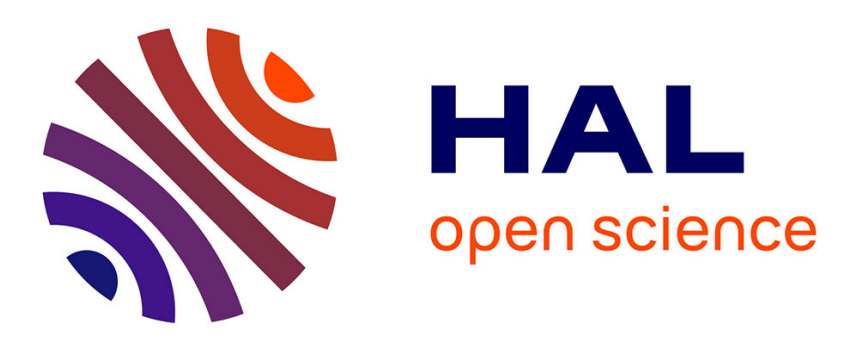

\title{
Random Sampling: Spectrum of Fluid Measured by Doppler Velocimetry
}

Abdeldjalil Ouahabi, Claude Depollier, Laurent Simon, Denis Kouamé, Marc

Lethiecq

\section{- To cite this version:}

Abdeldjalil Ouahabi, Claude Depollier, Laurent Simon, Denis Kouamé, Marc Lethiecq. Random Sampling: Spectrum of Fluid Measured by Doppler Velocimetry. IEEE International Instrumentation and Measurement Technology Conference 1996, IEEE, Jun 1996, Brussels, Belgium. pp.531-536, 10.1109/IMTC.1996.507439 . hal-03153381

\section{HAL Id: hal-03153381 \\ https://hal.science/hal-03153381}

Submitted on 26 Feb 2021

HAL is a multi-disciplinary open access archive for the deposit and dissemination of scientific research documents, whether they are published or not. The documents may come from teaching and research institutions in France or abroad, or from public or private research centers.
L'archive ouverte pluridisciplinaire HAL, est destinée au dépôt et à la diffusion de documents scientifiques de niveau recherche, publiés ou non, émanant des établissements d'enseignement et de recherche français ou étrangers, des laboratoires publics ou privés. 


\title{
Random Sampling: Spectrum of Fluid Measured by Doppler
}

\author{
A. Ouahabi*, C. Depollier ${ }^{\star \star}$, L. Simon ${ }^{\star \star}$, D. Kouamé ${ }^{\star}$, M. Lethiecq* \\ * LUSSI, GIP ULTRASONS / EIT 7 avenue M. Dassault 37004 Tours Cedex FRANCE \\ Phone: (+33) 47711226 Fax: (+33) 47289533 E-mail: ouahabi@balzac.univ-tours.fr \\ ** LAUM Avenue O. Messiaen 72017 Le Mans Cedex FRANCE \\ Phone : (+33) 43833270 Fax: (+33) 43833520 E-mail: depol@laum.univ-lemans.fr
}

\begin{abstract}
Spectral analysis of randomly sampled signals is discussed with reference to fluid velocity measured by ultrasonic (or laser) Doppler velocimetry. This study summarizes the merits, in the mean square sense, of the polygonal hold vs. the zero-order hold interpolator. The comparison is done for different sampling schemes, such as Poisson sampling. It can be shown that if the signal is sampled randomly with a Poisson, the output signal is equal to the original signal plus an uncorrelated additive white noise. Our purpose is to reduce the effect of this noise by using a simple first-order (or polygonal) interpolator. Similar results can be derived for uniform sampling with some missing samples or with time jitter.

Finally, we use a suboptimal method for detection of sampling jitter based on the bispectrum of sampled data
\end{abstract}

\section{INTRODUCTION}

Spect pectral analysis of digitized data conventionally requires the data to be equally spaced. It is well known that estimates of power spectra of fluid velocities have been made from data sets which are fundamentally unequally spaced. Generally these data sets are assumed to be equally spaced, or some preprocessing is performed in an attempt to produce an equaliy spaced data set from the raw, unequally spaced set.

In many situations where random signals occur it is of interest to form estimates of power spectra by processing digital samples. Frequently one has control over the way in which these samples are acquired. It is then easy to sample at equally spaced intervals of time, since the resulting data can be processed by well known methods, usually based on the fast Fourier transform (FFT) or parametric algorithms, to yield spectral estimates.

Situations do occur in practice, however, where it is not possible to dictate the times at which samples of a random signal can be acquired.
One such case is the measurement of the fluid velocity by the power spectral density using Doppler techniques. This situation is complicated by the random, intermittent nature of the velocity signal caused by the random arrival of particles at the measurement volume. Random sampling also occurs in other conditions, either voluntarily to use less memory, reduce processing or reduce bandwidth for digital transmission, or as a consequence of the nature of the measuring system.

Although random sampling may be more natural or efficient than uniform sampling it has not been used for several reasons. First, the nonuniform sampling theory is not simple. Second, the time and frequency analyses are rather involved. Finally, there is no simple and practical reconstruction method for error free recovery asopposed to low pass filtering in the uniform sampling case. In general, a nonlinear and/or time varying filter is required for exact recovery.

Recently [1], [2], the power spectral of an LDV (Laser Doppler Velocimetry) velocity signal was estimated by sampling at the arrivals of valid signal bursts and holding the values until another valid signal arrives. Unfortunately, the measured spectrum is filtered at the mean sample rate and it contains a filtered white noise spectrum caused by the steps in the sample and hold (zero-order interpolator) signal. It is possible to show that the power spectrum of the random samples consists of the power spectrum of the original signal plus additive noise.

Our problem is to reduce the effect of this noise by using a simple first-order (or polygonal) interpolator .

The main objectives of the present general study can be summarized as follows :

In section II, we briefly compare various reconstruction procedures: the zero-order hold, the exponential hold, the polygonal hold, and a linear nonrealisable interpolation procedure. In the next section, we consider the effect of random sampling in the case of uniform or Poisson sampling: the spectral density of random samples are hence derived explicitly.

Finally, we propose a way to passively identify the existence of such errors. The proposed test can be 
used to decide, given any sequence of data, whether timing errors in the sampling process or random sampling were present or not.

\section{MEAN SQUARE COMPARISON OF VARIOUS INTERPOLATORS}

Suppose that a random signal $x(t)$ is sampled at the sampling times $t_{n}$, thus leading to a set of data $\left\{x\left(t_{n}\right)\right\}_{n=0 . \pm 1 \pm 2 \ldots}$. The problem of optimum interpolation consists of finding an operation $M_{t,\{t h\}}$ such that

$$
y(t)=M_{t,\left\{t_{n}\right\}}\left[\left\{x\left(t_{n}\right\}\right]\right.
$$

is the "best" recovery of $x(t)$, for all $t$.

\section{A. The Zero-Order Hold Case}

$$
y(t)=x\left(t_{n}\right), \quad t_{n}<t<t_{n+1}
$$

This is the classical stepwise reconstruction procedure. It is easily seen that

$$
\begin{aligned}
& E[y(t)]=E[x(t)] \\
& \text { and } \\
& E\left[y^{2}(t)\right]=E\left[x^{2}(t)\right]
\end{aligned}
$$

In other words, $y(t)$ and $x(t)$ have the same mean and variance.

The mean-square error is

$$
\begin{aligned}
\overline{\varepsilon^{2}} & =E\left[\{y(t)-x(t)\}^{2}\right] \\
& =2 R_{x}(0)-2 \int_{0}^{\infty} R_{x}(\sigma) g_{1}(\sigma) d \sigma
\end{aligned}
$$

where $g_{l}(\sigma)$ denotes the probability density function associated with the random variable $L_{l}(l)$ (see Fig. 1) and $R_{x}(\sigma)$ is the correlation function of the original signal $x(t)$.

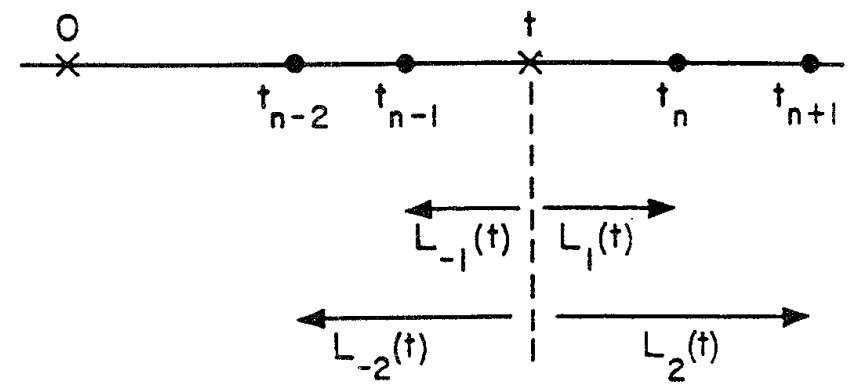

Notice that in the case of periodic sampling, the random variable $L_{. l}(t)$ is uniformly distributed in the interval $[0, T]$ where $T$ denotes the sampling period.

Poisson sampling: If $\lambda$ denotes the average number of sampling points per unit time interval, then

$$
g_{\mathrm{l}}(\sigma)=\lambda e^{-\lambda \sigma}
$$

Therefore, using (4), the mean-square error is

$$
\overline{\varepsilon^{2}}=2 R_{x}(0)-2 \lambda \int_{0}^{\infty} R_{x}(\sigma) e^{-\lambda \sigma} d \sigma
$$

\section{B. The First-Order (Polygonal) Hold Case}

The polygonal hold reconstructor defines the approximation function $y(t)$ as

$$
\begin{gathered}
y(t)=\frac{\left(t-t_{n}\right) x\left(t_{n+1}\right)+\left(t_{n+1}-t\right) x\left(t_{n}\right)}{t_{n+1}-t_{n}}, \\
t_{n}<t \leq t_{n+1}
\end{gathered}
$$

It is easy to see that $y(t)$ and $x(t)$ have the same mean values

$$
E\{v(t)\}=E\{x(t)\}
$$

and the mean-square reconstruction error is

$$
\begin{aligned}
\overline{\varepsilon^{2}}= & R_{x}(0)+R_{x}(0) E\left\lfloor\frac{L_{1}^{2}+L_{-1}^{2}}{\left(L_{1}+L_{-1}\right)^{2}}\right] \\
& +2 E\left\lfloor\frac{\left[L_{1} L_{-1} R_{x}\left(L_{1}+L_{-1}\right)\right.}{\left(L_{1}+L_{-1}\right)^{2}}\right\rfloor \\
& -2 E\left\lfloor\frac{L_{1} R_{x}\left(L_{-1}\right)+L_{-1} R_{x}\left(L_{1}\right)}{\left(L_{1}+L_{-1}\right)}\right\rfloor
\end{aligned}
$$

Notice that in the case of periodic sampling, the random variable $L_{I}$ is uniformly distributed in the interval $[O, T]$ and $L_{1}+L_{.1}=T$.

For Poisson sampling:, expression (7) becomes

Fig. 1. Sampling instants 


$$
\begin{aligned}
\overline{\varepsilon^{2}}= & R_{x}(0)+2 \lambda^{2} R_{x}(0) \int_{0}^{\infty} \int_{0}^{\infty} \frac{\sigma^{2}}{(\sigma+\beta)^{2}} e^{-\lambda(\sigma+\beta)} d \sigma d \beta \\
& +2 \lambda^{2} \int_{0}^{\infty} \int_{0}^{\infty} \frac{\sigma \beta}{(\sigma+\beta)^{2}} e^{-\lambda(\sigma+\beta)} R_{x}(\sigma+\beta) d \sigma d \beta \\
& -4 \lambda^{2} \int_{0}^{\infty} \int_{0}^{\infty} \frac{\beta}{(\sigma+\beta)} e^{-\lambda(\sigma+\beta)} R_{x}(\sigma) d \sigma d \beta
\end{aligned}
$$

\section{Performances of interpolators}

Performances of Poisson sampling are plotted in figure 3 for various interpolators when the input signal is a band-limited white noise [3]. Comparing the simplest interpolators, we can conclude that the first-order hold is much better than the zero-order hold.

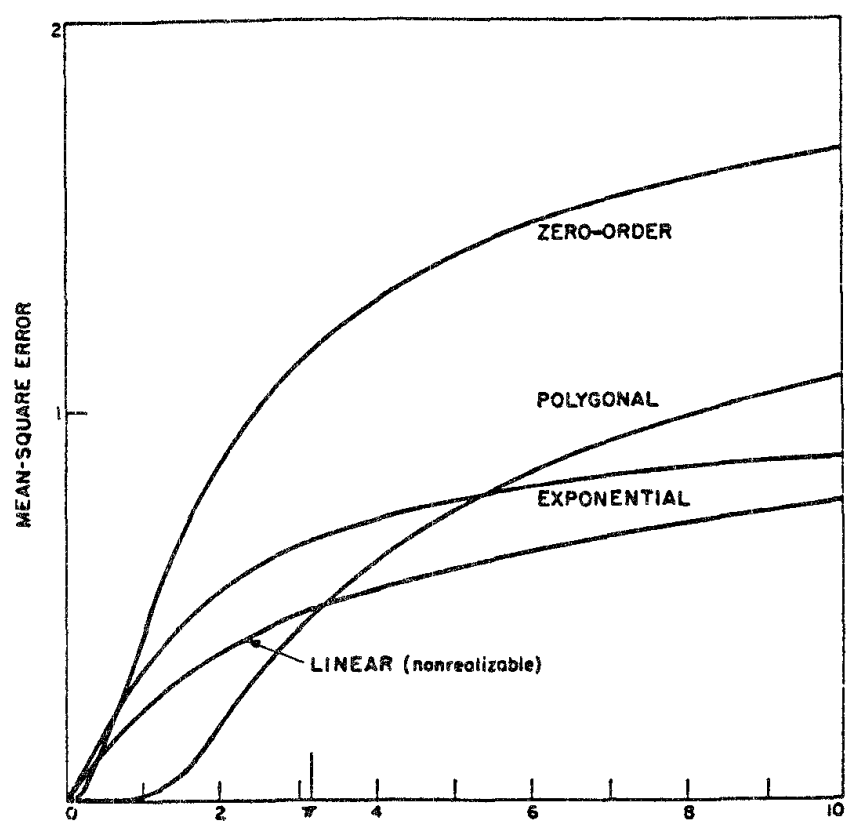

Fig. 3. Comparison of Various Interpolators: Poisson Sampling of a Band-limited Signal

\section{SPECTRAL ANALYSIS OF RANDOM SAMPLES}

\section{A. Definition}

It is well known that the spectrum of a periodic impulse train is another periodic impulse train in the frequency domain. However, the spectrum of a set of a random (or nonuniform) impulses (fig. 4), in general, is not another set of impulses. This implies that the spectrum of random samples might be totally smeared (aliased) in the frequency domain and we cannot use any linear timeinvariant filter for signal recovery.

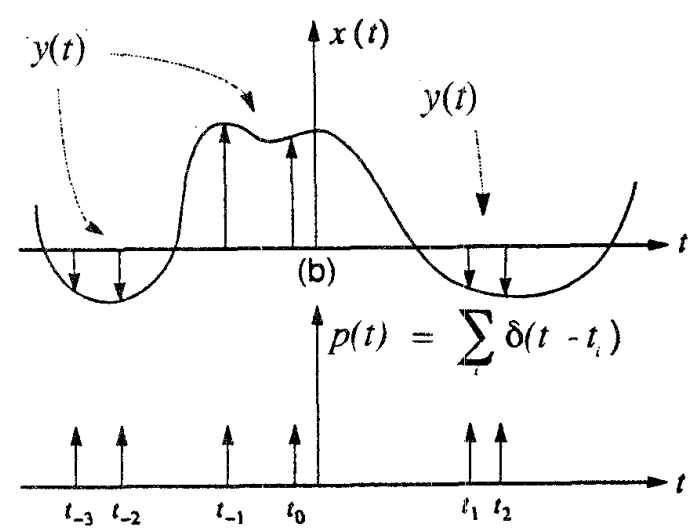

(a)

Fig. 3. (a) Sequence of Impulses Occurring at the Random Times $t_{i}$.

(b) Random sampling process.

If $p(t)=\sum_{i} \delta\left(t-t_{i}\right)$ is any random sequence of impulses and $x(t)$ is the original signal then the sampled signal is

$y(t)=p(t) . x(t)$.

Therefore, the power spectrum of the randomly sampled signal is

$$
S_{y}(f)=S_{p}(f) * S_{x}(f)
$$

where * is the convolution operator.

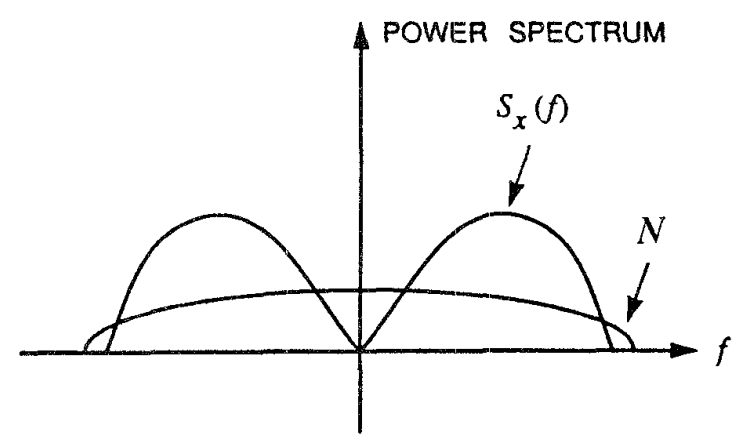

Fig. 4. Power Spectrum of Random Samples

For the case of Poisson impulsive sampling, the power spectrum of the point process, $p(t)$, is [11]

$$
S_{p}(f)=\lambda+\lambda^{2} \delta(f)
$$

where $\lambda$ is the average number of samples per unit time.

Therefore, substituting equation (10) into (9), the power spectrum of the sampled signal is

$$
S_{y}(f)=\lambda^{2} S_{x}(f)+N
$$

where $N$ equals $\lambda R_{x}(O)$.

The power spectrum of random samples (Poisson impulsive sampling) of a random signal resembles that 
of the signal imbedded in "white" noise as shown in figure 4.

Thus in the frequency domain, we generally expect the original signal to be corrupted by noise

\section{B. Discussion on Performances}

Equation (11) confirms the intuitive result that by increasing the density of sampling rate, the SNR (signal to noise ratio) improves. Papoulis [10] shows that if the sampling instant $t_{i}$ is a Poisson point process with average density $\lambda$, then the Fourier transform of the randomly sampled signal (if his energy is finite) is unbiased as $\lambda \rightarrow \infty$.

Masry [5] considers general spectral estimates (using the periodogram algorithm), with classical spectral windows (Bartlett, Tuckey and Parzen), based on finite number of observations taken at Poisson sampling instants.

He derives the asymptotic bias and variance of the estimate and shows that the bias is directly proportional to the bandwidth and the variance is inversely proportional to the bandwidth.

This behaviour is identical to that of the continuous-time estimates and seems comforting

A somewhat unexpexted result is the effect of the average sampling rate on the bias and variance of the estimate: the bias is independent of $\lambda$ and the variance is proportional to

$$
\lambda\left(S_{x}(f)+\frac{R_{x}(0)}{\lambda^{2}}\right)
$$

and consequently for each fixed frequency $f$, there is an optimal sampling rate $\lambda_{0}=\lambda_{0}(f)$ which minimizes the mean square error; with,

$$
\lambda_{0}(f)=\frac{R_{x}(0)}{S_{x}(f)}
$$

so that $\lambda_{0}$ is inversely proportional to the normalized value of the the spectral density.

\section{Interpolation}

There are several reconstruction methods. We mention only the most promising ones, namely, the non-linear technique, the iterative technique and the first order interpolator.

Marvasti [6] shows that the non-linear technique improves signal to noise ratio compared to simple lowpass filtering. Wiley [12] proposed an iterative procedure which can recover a signal from a set of nonuniform pulses without any distorsion after infinite iterations.

Based on the results obtained in section 11 , we assert that a simple first-order interpolator gives a good estimation of the power spectrum of the original signal without significant additive operations.
Recently, these good performances of the first-order interpolator were confirmed [9] practically for LDV application. Indeed, the theory valid [1], [2] for low fluctuation intensity flows shows that the measured spectrum is filtered at the mean sample rate and that it contains a filtered white noise spectrum caused by the steps in the sample and hold signal (obtained by a zeroorder interpolator). The effect of this additive noise is significantly reduced by a first-order interpolator especially at high frequencies where the distorsion is lower than for zero-order "sample and hold" processor .

\section{JITTER DETECTION}

\section{A. Jitter and Aliasing}

Sampling processes are rarely perfect - random clocktiming errors (jitter) are present in any practical clock used for sampling time. These errors cause aliasing which can have a considerable effect on the performance of discrete signal-processing algorithms Hinich [4] propose a test for aliasing using bispectral analysis applied in social science.

Aliasing can be especially serious for social science time series applications, since the sampling rate determined according to logistic and cost considerations without regard for the spectral characteristics of the underlying process. Once a sampling process is used to collect data, it is of value to test the observations for the presence of aliasing.

In engineering and physical-science applications, aliasing is usually avoided by filtering the signal to eliminate its energy above a certain cutoff frequency and then sampling the filtered signal at or above twice the cutoff frequency.

Consequently, in this paper we assume that in the absence of jitter, no aliasing exist

Based on Hinich's results, we attest that, while the discrete bispectrum of a uniformly sampled third-order stationary signal is zero in a triangle that is a proper subset of the principal domain [4], [5], [8] and [10], it differs from zero in the presence of jitter or random sampling. Exploiting this effect, we use a test for detection of sampling jitter, based on the bispectrum.

\section{B. Bispectrum and Test for Aliasing}

The discrete bispectrum of the uniformly sampled bandlimited, real, zero-mean and stationary process $x(k)$. satisfies

$B\left(\Omega_{1}, \Omega_{2}\right)= \begin{cases}\frac{1}{T^{2}} B_{c}\left(\frac{\Omega_{1}}{T}, \frac{\Omega_{2}}{T}\right), & \text { on IT } \\ 0, & \text { on OT }\end{cases}$

where IT and OT are the inner triangle and the outer triangle of the principal domain (the triangle $O A B$ of Fig. 5), respectively, and $B_{C}$ is the two-dimensionnal Fourier 
transform of the third moment [8], [10]. $R(m, n)$ defined by

$$
E\{x(k) x(k+m) x(k+n)\} \text {. }
$$

The suboptimal test, which decides $\mathrm{H}_{0}$ or $\mathrm{H}_{1}$, on the basis of the bispectrum estimates is

$$
\begin{aligned}
& H_{0}: B\left(\Omega_{1}, \Omega_{2}\right)=0 \\
& \forall\left(\Omega_{1}, \Omega_{2}\right) \in \text { OT } \Rightarrow t_{n}=n T \quad \text { (no jitter) } \\
& H_{1}: B\left(\Omega_{1}, \Omega_{2}\right) \neq 0 \\
& \forall\left(\Omega_{1}, \Omega_{2}\right) \in \text { OT } \Rightarrow t_{n}=n T+\varepsilon_{\mathrm{n}} \quad \text { (jitter) }
\end{aligned}
$$

where $T$ is the sampling period and $\left\{\varepsilon_{n}\right\}$ are i. i. d. random variables.

Notice that the only prior knowledge required concerning the continuous process is that it is band limited and it has a nonzero bispectrum.

This test is similar to the tests in [4], [5] and [8], which were used for detection of non-Gaussian or nonstationary signals.

It is possible to exploit this suboptimal test, using standard statistic approximations and deriving sufficient condition for high probability detection. The proposed test is made in the bispectral domain, and not that of the data itself, therefore it is suboptimal. However, the test is robust, independent of the signal statistics, and applicable for random sampling of any statistical distribution.

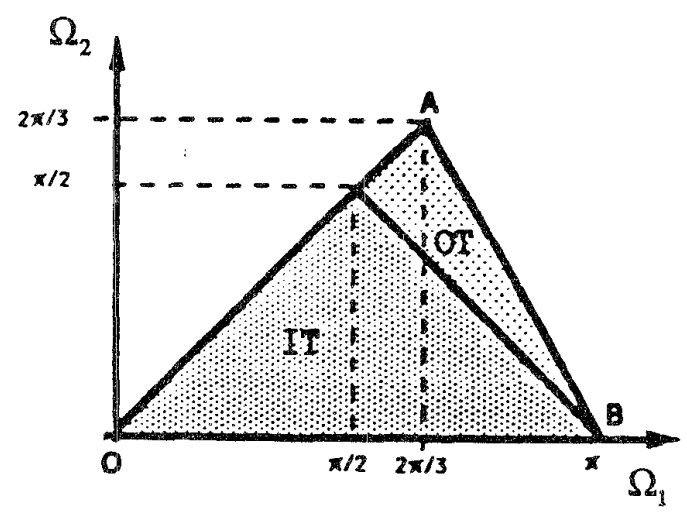

Fig. 5. The bispectrum principal domain

\section{SUMMARY AND CONCLUSIONS}

Spectral analysis is more difficult in the case of unequally spaced data than in the case where the data are sampled at equal intervals.

There are a number of open theoretical questions, for example regarding the nature of aliasing in estimates from an arbitrary sampling scheme, the exact analytic expression of the power spectrum in the case of a firstorder interpolator and the correct means of model identification for parametric estimates. There are a number of different methods available, but little practical experience to show their relative merits and assist in choosing which should be applied to a particular problem.

We have investigated the power spectrum estimation from randomly sampled signals and obtained preliminary results assuming that the sampling times are Poisson distributed, with a constant average sampling rate. Indeed in the majority of practical situations this distribution will be a good model. Moreover, we show that if the point process has a Poisson probability distribution then the additive noise is uncorrelated to the input signal and its power spectrum is proportional to the total signal power.

Therefore, the estimation of the power spectrum is improved in the mean square sense without significant additive computations (compared to the zero-order interpolator), when the interpolator is linear (first-order hold) and the sampling rate (the average number of samples per unit time) is at two or more times the Nyquist rate.

We have also proposed to use the bispectrum on OT for detection of random sampling : given samples of an unknown band-limited signal who has nonzero bispectrum, we can tell wether the sampling clock has jitter by looking at the bispectrum of the sampled data. It is done by applying a binary hypothesis test, whose outcome decides wether the bispectrum on OT is zero or not.

Although, this study was motivated primarily by the need to analyze the experimental information mentioned above $[9.11]$, the ideas upon which it is based are of a fundamental nature and as such are likely to have a wide area of applications: communications, control, biomedical, geophysics...

\section{REFERENCES}

[1] R. J. Adrian and C. S. Yao "Power spectra of fluid velocities measured by laser Doppler velocimetry," Exp. in Fluids, vol. 5, pp. 17-28, 1987

[2] L. Boyer and G. Serby, "Random sampling: distorsion and reconstruction of velocity spectra from fast Fourier transform analysis of the analog signal of laser Doppler processor," J. Appl. Phys., vol. $60, n^{2} 8$. pp. 2699-2707, 1986.

[3] F. J. Beutter, O. A. Z. Leneman, "The spectral analysis of impulse processes, "Information and Control, vol. 12, pp. 236-258, 1968.

[4] M. J. Hinich, "Testing for Gaussianity and linearity of a stationary time series," J. Time Seris Anal., vol 3, pp. 169-176.1982

[5] M. J. Hinich and M. A. Wolinsky, "A test for aliasing using bispectral analysis," J. Amer. Statist. Assoc., vol. 83, n. 402. pp. 499-502, 1988.

[6] F. A. Marvasti A unified approach to zero-crossing and nonuniform sampling of singte and multidimensional signals and systems. Nonuniform Publications, Oak Park, IL, 1987.

[7] E. Masry, D. Klamer and C. Mrabile, "Spectral estimation of continuous-time processes: performance comparison between periodic and poisson sampling schemes, "IEEE Trans. on Automatic Control, vol. $\mathrm{AC}-23, n^{\circ} 4$, pp $679-685,1978$. 
[8] C. L. Nikias and M. R. Raghuveer," Bispectrum estimation: a digital signal processing framework," Proc. IEEE, vol. 75, pp. 869-892, 1987.

[9] A. Ouahabi, C. Depollier, L. Simon, D. Kouamé, M. Lethiecq, "New results in spectrum analysis of randomly sampled processes", Proc. of the I. Conf. Modelling, Identification and Control, Innsbruck, feb.1996

[10] A. Papoulis, Probability, Random Variables, and Stochastic Processes, Mac Graw-Hill (Third Edition), N. Y., 1991.

[11] L. Simon, J. Scholten, J. Fitzpatrick, "Amelioration du traitement des données expérimentales par interpolation d'ordre 1," CFM Strasbourg. sept. 1995.

[12] R.G. Wiley, "On an iterative technique for recovery of bandlimited signals," Proc. IEEE, pp. 522-523, 1978. 